\title{
Hermann Grid Optical Illusion and The Rebelling Dots of Reality
}

\author{
Author: \\ Krishanu Kumar Das \\ Affiliation of the Author: \\ Department of Medicine, HLG Memorial Hospital, Asansol, India
}

E-mail Address:

dr.krisdas@yandex.com 


\begin{abstract}
:
In 1870, Ludimar Hermann reported an optical illusion that has been popularized thereafter after his name. In this Hermann Grid illusion, we see either white grid upon the background of complete black or black grid on the background of complete white. If we watch the grid, we see blobs of darkness or blobs of white smudges appearing at the intersections of the white or black grid respectively that are more prominent at peripheral view. A number of approaches to explain this illusion have been ventured in course of time. In this article, we will take the endeavor to explain it from the angle of familiarity with the previous associated memories of grid views. To summarize our view, we can say that we see Hermann grid illusion because of the impossibility of the Hermann grid in reality.
\end{abstract}

Keywords: Optical Illusion, Hermann Grid, Associative Memory, Visual perception. 


\section{Introduction:}

In 1870, Ludimar Hermann reported an optical Illusion which is popularly known as Hermann Grid Illusion. This illusion is illustrated as a white grid view on the background of completely black darkness. If we look at the grid, we see blobs of darkness appear at the intersections of the white grid, that dissipate along the grid-bars also. The Illusion is not seen in focal view, but prominent at the peripheral view. Thereafter, Hering observed that the illusion is also visible in grid of opposite polarity - that is blobs of white smudges appear in the intersections of black grid against the white background [from Spillmann, 1994]. Later, it was found that Hermann grid illusory effects are also visible in case of contrasting chromatic grids. This illusion has invited immense attention till date because of the apparent hardships that are encountered to explain this illusion. Many approaches have been emanated from different authors to explain this illusion in course of time. In the later years, Bergen forwarded another variant of the illusion through low filter pass where the bars of the grid were smeared with darkness or greyish tinge against the more luminant intersections [Bergen, 1985]. This variant was further worked upon by Schrauf et al., and popularly known as scintillating grid illusion [Schrauf et al., 1997]. In this illusion, we see blobs of darkness appear only in the intersections, but not in the bars (Figure 1).

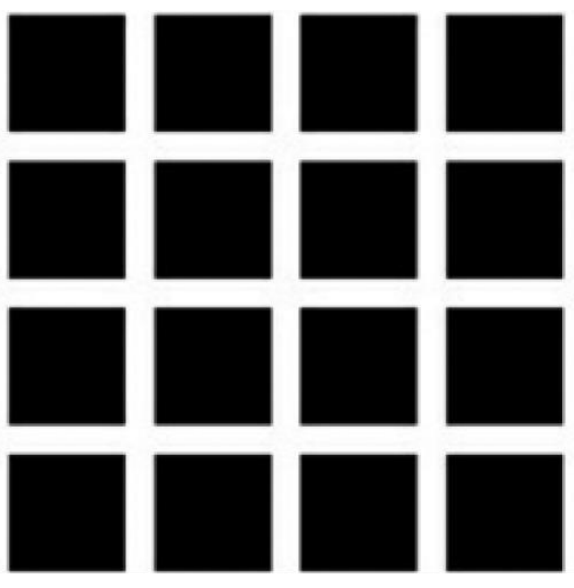

(A)

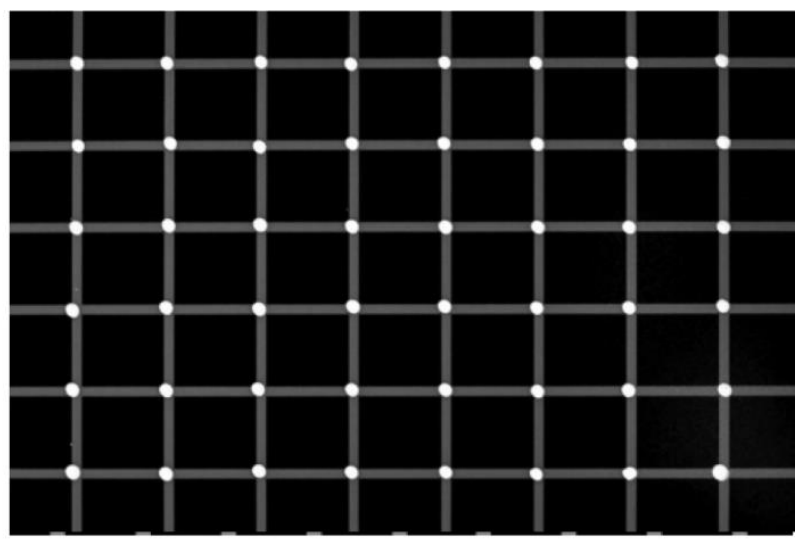

(B)

Figure 1. A) Hermann-Grid illusion. Darkness appears at the intersections of the grid which dissipates also along the borders. B) Scintillating grid illusion. Dark dots appear and disappear at the intersections.

Classical explanation of Hermann Grid illusion was proposed by Baumgartner [Baumgartner, 1960]. According to this theory, illusory effect is generated by the response of retinal ganglion cells with concentric on-off or off-on receptive fields (due to lateral inhibition). Where there is more light surrounding the intersections, central cells are more inhibited by adjacent receptor cells, than what happens at the bands (Figure 2). But this theory has not been widely accepted because of its inconsistencies. When the grid is tilted, or the grid bars are made curved or 
distorted [Geier, 2008], the illusion disappears, though the same mechanism is applicable to those cases also for creating the same illusion.
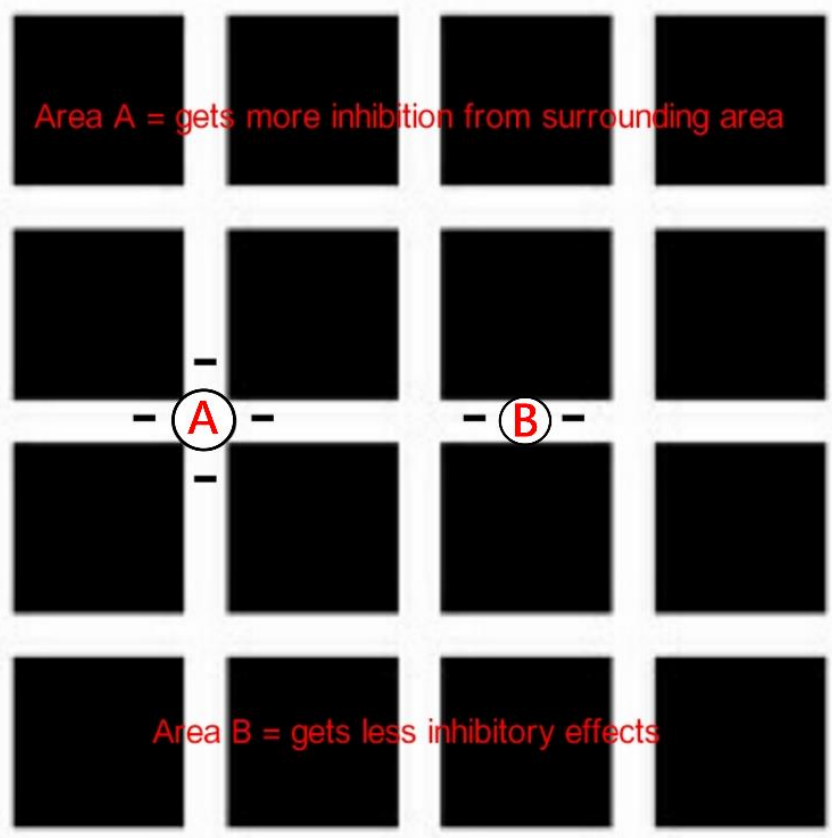

Figure 2. Classical explanation of Hermann grid illusion.
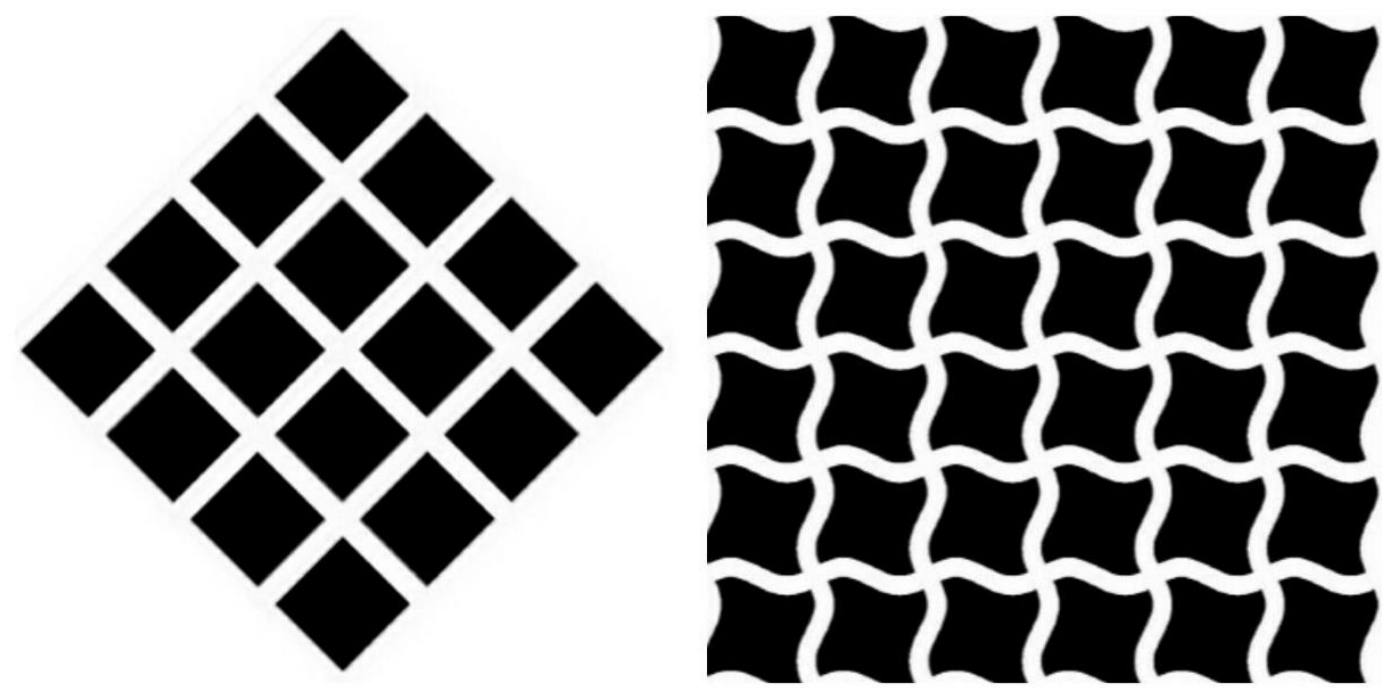

Figure 3. Tilted and Curved-barred grid. 
Schiller and Carvey proposed an alternate theory - "S1 simple cell theory" which suggests that the illusion results from the S1 simple cells in the primary visual cortex (V1), which are orientation specific. These S1 simple cells are excited either by ON type of receptive fields or by OFF type of receptive fields in retina, and they may be called as ON S1 cells or OFF S1 cells. According to them "illusory smudges are the result of the relative degree of activity of the ON and OFF S1 cells at the intersections, as compared with activity at non-intersecting locations" [Schiller \& Carvey, 2005]. But why one observes illusory spots in the middle of the intersections - their explanation leaves this question unanswered [Geier, 2008]. Of course, same question is applied to Baumgartner's theory also. At the end of the article, in figure 12, we can see the effects of lateral inhibition on bars. Here, the darkness starts to span from the margin of contrasting border.

\section{Explanation of Peripheral Vision and Illusion:}

Mind does not always see what eyes see. Only 10-20\% of the inputs to the lateral geniculate nucleus comes from retina, other inputs come from other regions of the brain including feedback inputs from the visual cortex. In visual cortex, the number of neurons concerned with vision is 1000 times the number of fibers in the optic nerve [Ganong, 2019]. There are at least 25 visual areas that have been identified in non-human primates, that occupy over half of their neocortex; and retinotopic mapping through non-invasive fMRI in human has shown close resemblances of visual areas to those of the monkeys [Sereno et al., 1995]. What we see partly depends on what we want to see or what we anticipate to see. And this anticipation is generated based upon our previous experiences and knowledge, or more precisely to say, all our associated memories attached with it. Associated memories (also called associative memories) mean sets of memories associated with different objects and situations. Retrieval of these associated memories may be triggered reflexively. Optical illusions are more related to associated memories rather than any optical factors. In majority of cases, they take the route of deception through the reflexive retrieval of the firmly embedded associated memories. Whenever, we see an image, we cannot concentrate on every point of it. We usually concentrate our vision on a particular region which constructs focal or foveal view both in monocular and binocular vision and perceive the periphery partly through the overall interpretation of the image related with our knowledge using expectations or anticipations from our already formed assembled associated memories. There are two factors that mind always encounters in perception, 'the possibility' and 'the impossibility'. Our focal vision, where we concentrate our attention, challenges impossibilities; but our peripheral vision does not challenge impossibilities, rather lies between possibilities and impossibilities, if any there. In case of a firmly pre-embedded associated memory, our mind often perceives the image according to the pre-instituted memory and knowledge, rather than the actual image, particularly in peripheral vision. 
For example, if we look at the following image (Figure 4), we see lips in the face in place of eye. But if we concentrate on other parts of the image, or we do not concentrate on any point at all, we perceive eye, not lips because with that view we are not accustomed at all. Our peripheral view is partly built, or tends to be formed based upon our previously embedded associated memories or our previous experience. This is also a reason why peripheral vision is poorer in children than adults, because of poorer pre-embedded memories. And why pedestrian accident rate is higher in children than adults [David, 1986].
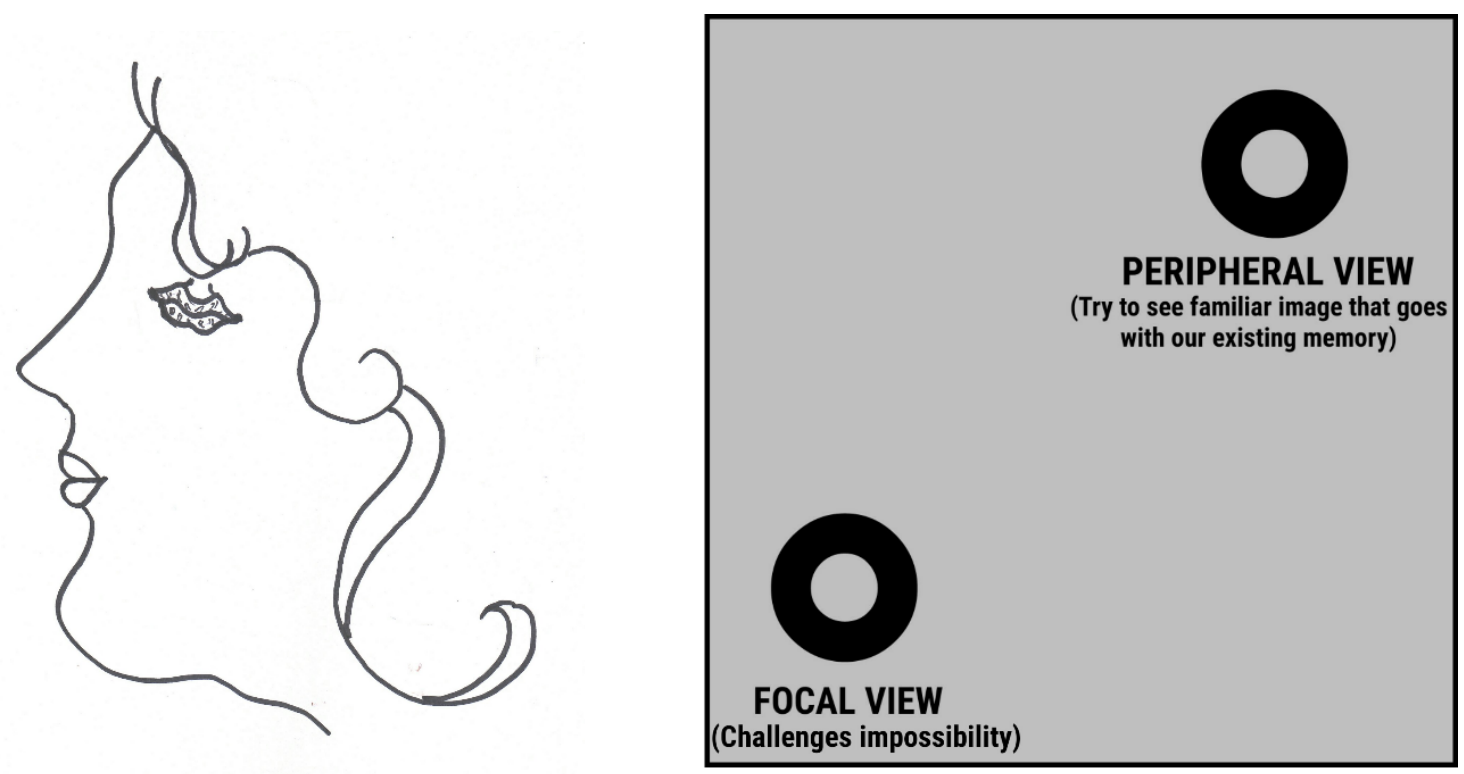

Figure 4. Eye-lips illusion (author)

Now in case of optical illusions generated through retrieval of associated memories, the strength of an optical illusion is directly proportional to the strength of associated memories. It is guided by the following formula:

If the strength of association between a given cue $M$ and illusory outcome $J$ is $A_{m, j}$, and the illusory strength of outcome $\mathrm{J}$ over cue $\mathrm{M}$ is $\mathrm{I}_{\mathrm{m}, \mathrm{j}}$, then -

$I_{m, j} \propto A_{m, j}$ (where the contextual variable is fixed).

(For estimation of associative strength, see literature cited in my previous article Das, 2017).

Here, the cue $\mathrm{M}$ is depended on both the focal view and the whole context of the image. And change of illusory strength $\Delta \mathrm{I}_{\mathrm{m}, \mathrm{j}}$ is proportional to change of associative strength $\Delta \mathrm{A}_{\mathrm{m}, \mathrm{j}}$. But it 
is significant to notice that when the real outcome is different, there is also simultaneous concurrent change of associative strength for a particular cue and outcome. So, in the next trial both the strengths will be changed and the change of illusory strength will proportionally follow the change of associative strength, maintaining contextual variable is fixed. This predicts the fact that repeated exposures of an optical illusion reduce the illusory effect of the illusion to a subject. For that, optical illusions are most successful in those cases, where associative strength is high, or where in normal life lost associative strength is recovered through regular experience at a much higher rate. Now, if we equate the above proportionality,

$\mathrm{I}_{\mathrm{m}, \mathrm{j}}=\tau \times \mathrm{C} \times \mathrm{A}_{\mathrm{m}, \mathrm{j}}$

$I_{m, j}=$ Illusory strength between a given cue ' $M$ ' and outcome ' $J$ ', when real outcome is different.

$A_{m, j}=$ Associative strength between given cue ' $M$ ' and outcome ' $J$ '.

$\tau=$ Illusory coefficient which is a constant at a specific region maintaining certain conditions.

$\mathrm{C}=$ Contextual Variable

Contextual variable $\mathrm{C}$ again is the multiplication of observer's variable ' $\mathrm{O}$ ' and situational variable ' $\mathrm{S}$ '.

$\mathrm{C}=\mathrm{O} . \mathrm{S}$

Observer's variable 'O' depends on the observer's emotional state, mood, and the amount of attention he or she is paying. Situational variable 'S' depends on light, shades, clearness of the medium and other situational determinants at the time of the illusion to be experiencing.

Now, maintaining the above principles, if illusory outcome is strong enough to overpower far above the real outcome or reality (we consider the real outcome is different), an illusion is generated. When illusory outcome and real outcome are nearly equal in strength, we see an image fluctuating or confusing in nature.

Illusory co-efficient is not a fixed constant. It is both sense organ specific and region specific. Illusory co-efficient is different in case of different senses - visual, auditory, gustatory, olfactory, tactile and proprioceptive. For visual perception, the illusory co-efficient for all cues of a particular color is constant at a particular receptive field ' $\mathrm{P}$ '. The receptive field represents the synaptic link-up of each ganglion cell with a number of receptor cells. Illusory coefficient is different in different receptive fields in different directions away from fovea. As the types and concentrations of retinal photoreceptor cells and other cells are not equally distributed in all directions from fovea, $\mathrm{P}$ is both distance and direction, i.e., region specific. Cone cells concentrations are maximum in fovea for highest visual acuity. Rods are absent in fovea; then 
their concentration gradually rises in parafoveal region to reach the peak at nearabout $20^{\circ}$ eccentricity along horizontal meridian. At the periphery, concentrations of both rods and cones are reduced as well as the number of ganglion cells is diminished too rendering a single ganglion cell invested for taking up information from larger area. So, the receptive field varies in sizes. In fovea, information from each cone cell is carried by a single ganglion cell making the image highly distinctive. Whereas in periphery more number of cells are taken up by a single ganglion cell making the size of the receptive field bigger as well as the quality of image blurred. So, if the receptive field $\mathrm{P}$ increases the distance from the posterior pole or fovea centralis, the illusory co-efficient also increases. More clearly to say, where in the retina the concentration of cells for real perception is less, the mind takes the course of imagination more from its pre-established knowledge. And the illusory co-efficient for that region increases. At the blind spot the illusory co-efficient is maximum as there is no cells for real perception, and at fovea the illusory co-efficient is minimum. In Figure 5, visual acuity in scotopic vision has been shown with respect to eccentricity along the horizontal meridian. If we consider white light, the graph of the illusory coefficient $(\tau)$ will be just the reverse of the graph of the visual acuity (VA).

Furthermore, the illusory coefficient of a particular receptive field is fixed for a particular color (red, green, blue, white). So, depending upon the distribution of four types of photoreceptor cells containing four classes of photopigments, illusory coefficient has also four values for colors in a particular receptive field. To elaborate a little, say, in a receptive field cone cells for detection of green light is absent. Now, if any green object is reflected on that receptive field, vision of that field would be highly illusory because of its high illusory coefficient for green light. That would not be so for other red, blue, or white colored light.

However, the above mentioned equations are the simplified mathematical equations to understand the overall process. In reality, the process is a little more complex. First of all, any given cue just does not depend on the focal view, it also depends on the overall image. With respect of this aggregation (focal view + overall image), the cue is determined and illusory perceptions are settled at different points according to above formula. This is responsible for Gestalt effect. Secondly, there are a few more optical phenomena that has to be encountered too when interpreting optical illusions. Most important of them are lateral inhibition and dark and light adaptations in case of normal eyes. Illusory coefficient would be different in photopic and scotopic vision because of differential adaptations and activities of rods and cones with different light intensities. If we consider pathologic conditions, then refractive errors, defects in accommodation, defects in color vision will also have to be considered. A myopic person without glasses would see more illusion at distant vision than a normal person. 


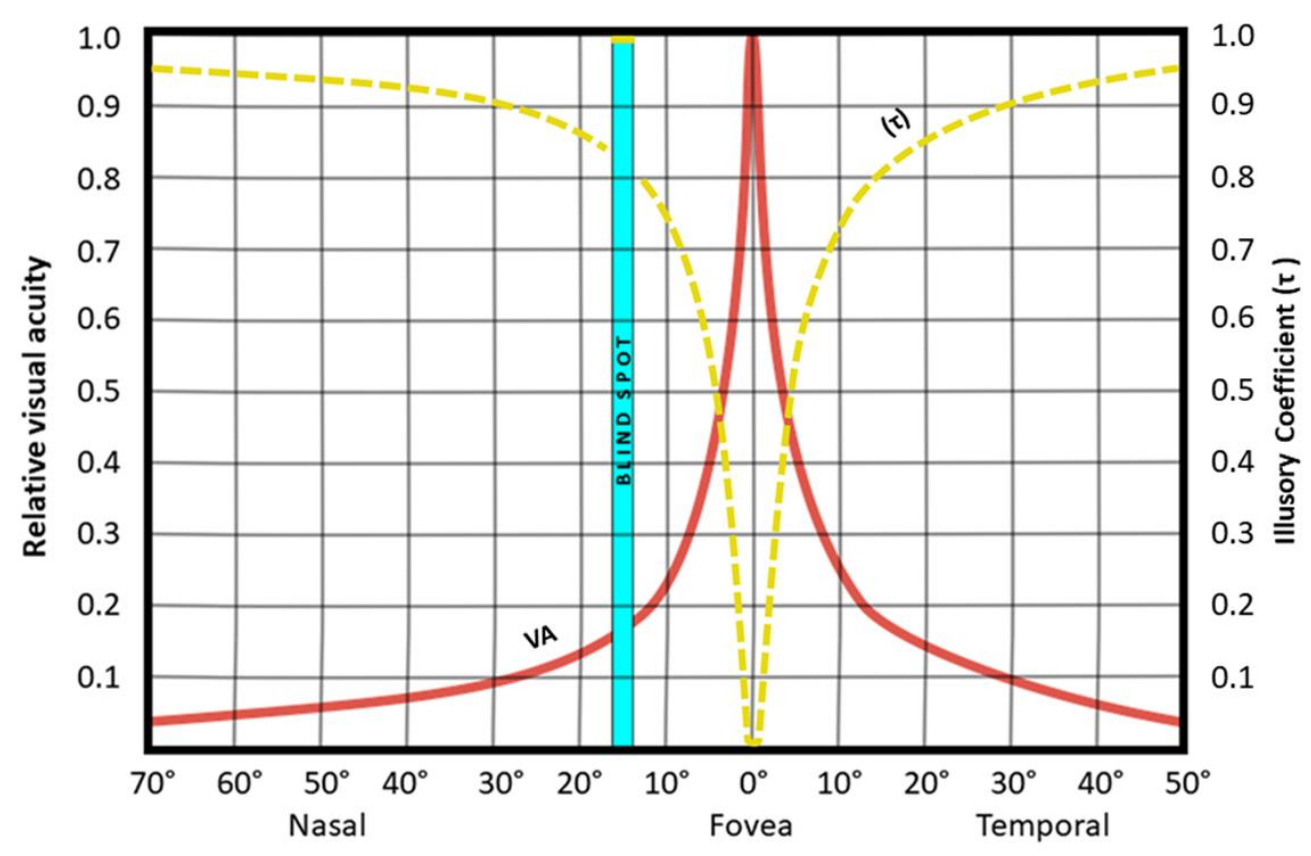

Degrees eccentric from fovea along horizontal meridian

Figure 5. Variations of visual acuity (VA) in the retina in photopic vision along horizontal meridian. (modified from Parsons' Diseases of The Eye, $22^{\text {nd }}$ ed., Editors: Sihota \& Tandon, 2015, Elsevier). In case of white light, the graph of illusory coefficient $(\tau)$ will be just the reverse of the visual acuity (VA).

\section{Superimposition in Peripheral Vision:}

Now, what happens if there is more than one possible outcome in a peripheral visual field considering the aggregated cue of focal view and context of the whole image?

We presume in the visual field point ' $F$ ' is the foveal or focal point. From $F$ at a particular distance and direction ' $\mathrm{P}$ ' is a receptive field in peripheral vision. For a particular cue $\mathrm{X}$ (depending on both focal view and context of the whole image), there are three outcomes possible at point $\mathrm{P}$. Let us say, they are $\mathrm{Q}_{1}, \mathrm{Q}_{2}$, and $\mathrm{Q}_{3}$. And their individual illusory strength with respect to illusory co-efficient of point $P$ are $i_{1}, i_{2}$, and $i_{3}$, respectively. Now, if the real outcome is different at the point $\mathrm{P}$, the illusory outcome at point $\mathrm{P}$ will present superimposition of three outcomes $\mathrm{Q}_{1}, \mathrm{Q}_{2}$ and $\mathrm{Q}_{3}$ in the following fractions.

Illusory Outcome $\sim\left(\mathrm{i}_{1} / \mathrm{i}_{1}+\mathrm{i}_{2}+\mathrm{i}_{3} \times \mathrm{Q}_{1}\right)+\left(\mathrm{i}_{2} / \mathrm{i}_{1}+\mathrm{i}_{2}+\mathrm{i}_{3} \times \mathrm{Q}_{2}\right)+\left(\mathrm{i}_{3} / \mathrm{i}_{1}+\mathrm{i}_{2}+\mathrm{i}_{3} \times \mathrm{Q}_{3}\right)$

If $\mathrm{n}$ number of outcomes are possible for the cue $\mathrm{X}$ at the receptive field $\mathrm{P}$, then total illusory outcome for cue $\mathrm{X}$ at point $\mathrm{P}$ will be: 
$\left.\sim\left(\mathrm{i}_{1} / \sum_{(\mathrm{k}=1 \text { to n })} \mathrm{i}_{\mathrm{k}} \times \mathrm{Q}_{1}\right)+\left(\mathrm{i}_{2} / \sum_{(\mathrm{k}=1 \text { to n })} \mathrm{i}_{\mathrm{k}} \times \mathrm{Q}_{2}\right)+\ldots .+\mathrm{i}_{\mathrm{n}} / \sum_{(\mathrm{k}=1 \text { to n })} \mathrm{i}_{\mathrm{k}} \times \mathrm{Q}_{\mathrm{n}}\right)$

\section{Now what happens in case of Hermann Grid illusion?}

Optical mechanisms are inadequate overall to explain Hermann Grid illusion. According to this study, the exact reason for this illusion does not lie on the aberration of optical mechanisms, but lie on the aberration in the translation of the optical stimuli to the visual perception in our brain.

We are very accustomed to gridded views. Not only looking through the gridded windows, but when we walk along the streets, views of blocks of houses, shops, posts, bars by the sides, with light or darkness coming through them, build our respective impressions strong through the absorbed memories of grid views. Neglecting the curvature, the earth's surface is horizontal, and on it gravitational force is perpendicular. For that, our world is more horizontal and vertical in nature than any other form. And this is the reason behind Hermann-Grid optical illusion.

In Hermann Grid illusion, we challenge 'the possibility' and 'the impossibility'. If the inside light is so bright to make the grid complete white, the darkness beyond the grid could not be as dark as complete black due to reflection and scattering of light by the grid. When we look at a bright light source, there appears a hallow around it due to scattering of light by the atmospheric molecules and particles. The darkness around the light source is smudged with same tinge of color. To watch background as dark as complete black, the insight should be less lighted or the grid should be darkish. So, the illusion appears at the peripheral vision which lies between the possibility and the impossibility. In scintillating grid illusion, the borders have already been smudged with darkness. So, the illusion does not appear at the borders as we are accustomed to see that type of darkness only through that colored grid.

In the vice versa case, if the background is bright white, bright yellow, bright red or of any bright color, and the grid is of different color, the illusion would also appear, because due to scattering of light, and to some extent reflection of the light from the inside objects, the grid will have a tinge of that color.

So, for theoretical purpose, for easier understanding, whether the background is dark or bright we can presume that background is scattering its own color to produce its effect on the grid, and that what happens in reality. In illusory view, depending upon the high illusory coefficient at the peripheral visual field and strong associative strength of the memories of grid, illusions appear at the periphery; and we see rebelling dots of reality saying, as if, we were here but you are evicting us from here.

Now, why the darkness or smudge (effect) is more prominent at the intersections? - It is because of overlapping areas of scattering halos (from four quarters) at the intersections in reality. Why we do not see the illusion when the grid is tilted at 45 degree or the shape of the 
grid is changed? - Because we are not accustomed to see those views. We don't see any more those as usual familiar views; we just see those as a design or a painting that does not challenge our memory.

To check the real view of the grid, I put a black grid against the daylight and photographed. When I closely observed the photograph, I got that the white smudges are there in the intersections that dissipated along the grid bars also. This is not illusion but reality. These are due to scattering of light by atmospheric molecules and particles over the dark areas. But the margins of the grid-borders appear comparatively dark. In my previous article, I presumed it is due to lateral inhibition. But lateral inhibition cannot affect the photograph, and it is prominent in photograph even after selective zooming. So, it is most probably due to overlapping of scattered light from the two sides in the middle of the bars making the margins of the bars less smudgy. And at the intersections, they get scattered light from four sides making those regions most greyish.

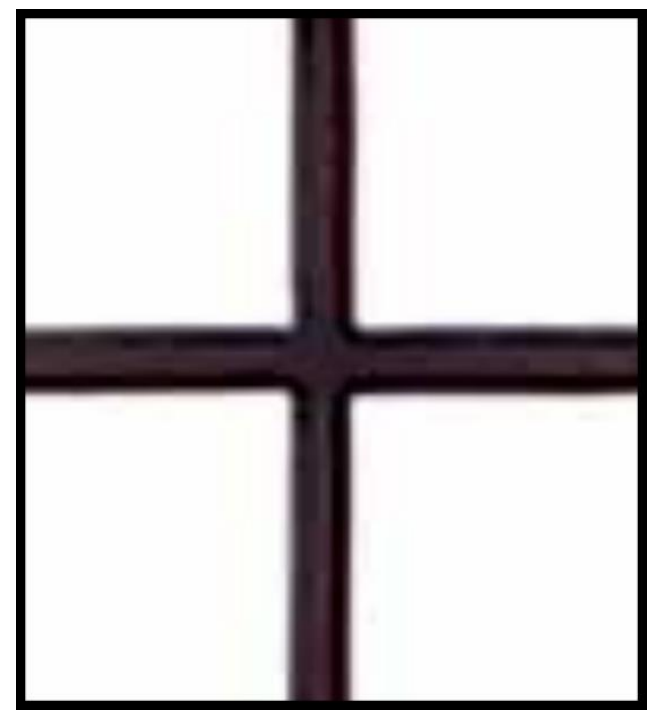

Figure 6. Grid in reality: Image is taken by 13-megapixel camera without zoom from the opposite direction of light at a distance of 2.5 meters from the grid by hand (not tripod), then the image has been cropped in photoshop. The grid is not complete black, it is brownish black, but completely monochrome and there are no white smudges within it. The width of the grid bar is $1 \mathrm{~cm}$ and it is cut from uniform thickness paper card.

Now we take, point $\mathrm{C}$ is the center of the grid junction, and is being illuminated by scattered light coming from the four quarters. From a fixed distance, whether the point $\mathrm{C}$ will be the highest luminous point of the grid junction, that depends on two factors - (1) distribution of scattered light intensity and (2) width of the grid bar. 
Scattered light intensity depends on i) composition of the medium (air) - that is properties (size, shape, reflectivity) and density of the particles in the medium; ii) intensity and wavelength of the incident light; and iii) the angle of scattering. But the distribution of scattered light intensity does not have a uniform value, as more light scattered and absorbed, less light enters into the successive layers. This light attenuation occurs in exponential fraction (Beer's law). If the intensity of the incident light is $\mathrm{I}_{\mathrm{O}}$, then after travelling through the $\mathrm{M}$ distance of the medium, due to scattering, attenuated light intensity $\mathrm{I}_{\mathrm{M}}$ will be:

$\mathrm{I}_{\mathrm{M}}=\mathrm{I}_{\mathrm{O}} \times \mathrm{e}^{-\mathrm{TM}}$

$\mathrm{T}$ is the turbidity (dependent on particles' properties and concentration) of the medium. For that, the chance of $\mathrm{C}$ point for being the highest luminous point of the grid junction increases when the width of the grid bar is less. This is true also for illusory case. But to observe the effect, the grid width has to have a minimal value that depends on subjective visual acuity. Consider the images in Figure 7. Here, the illusory effect is more visible in grid b, than grid a. Now if the observer increases the distance from the grid, volume of the scattering medium will increase. But light attenuation will occur according to the inverse square law. So, if the observer increases the distance from the grid, the effect (real and illusory) will increase, but it will be gradually blurred.
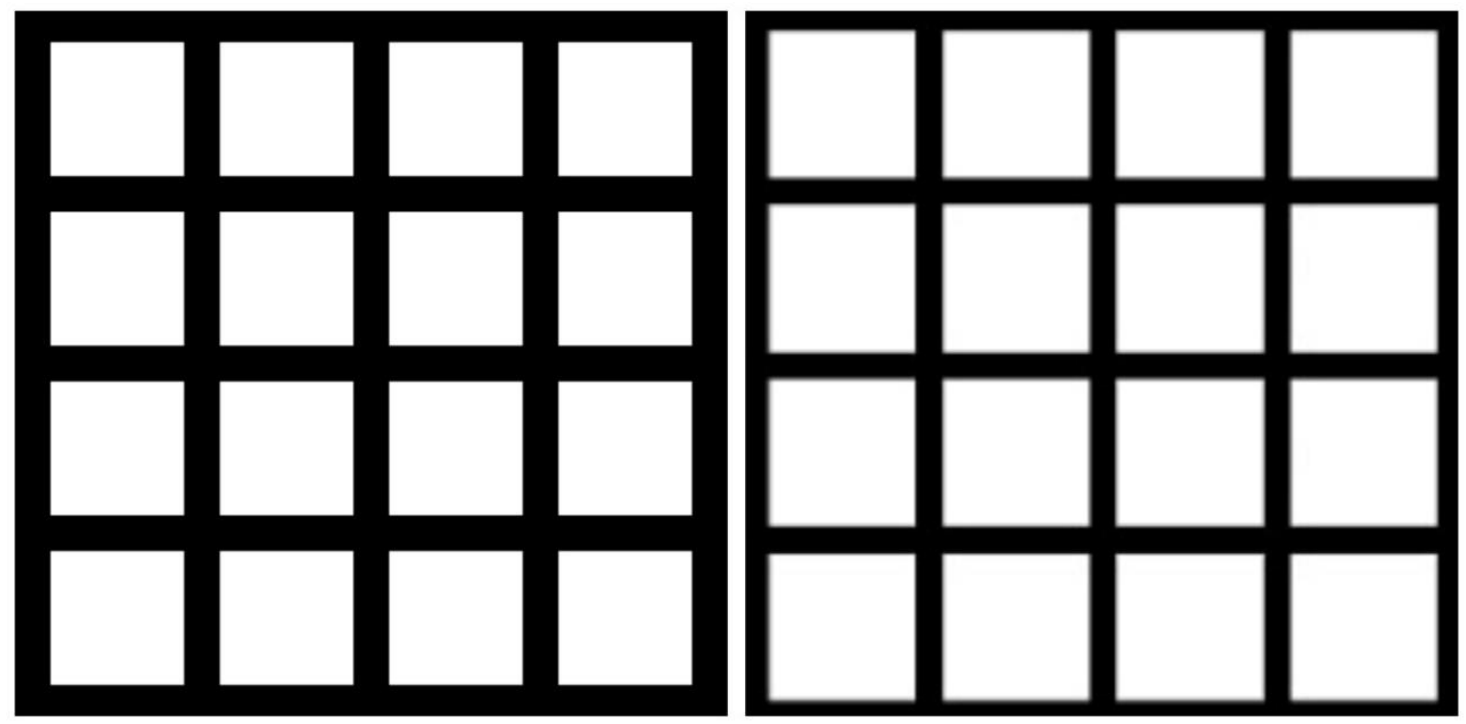

Figure 7. The illusory effect is more visible in grid $b$ than grid a. And if we look at the images from more distance, the illusory effect will further grow

\section{Further Support for This Theory:}

So, according to this theory, Hermann Grid illusion and its variants are depended on familiarity with the images, or on our previously embedded associated memories attached with the images. 
Here below, we present some more images that would further clarify that conception. In figure 8 , there are four images that evoke our familiar views of buildings or building-like structures. So, we can see the illusion (either darkness or white spots) at the intersections. In figure 9, there are four images that do not instigate our familiar views. And obviously, we do not see any illusions here as well. In figure 10, the upper half of the image coincides with our familiar views, and we see illusions there. But as we progress downwards along the image, the view recedes from our familiarity along with disappearance of illusion gradually.
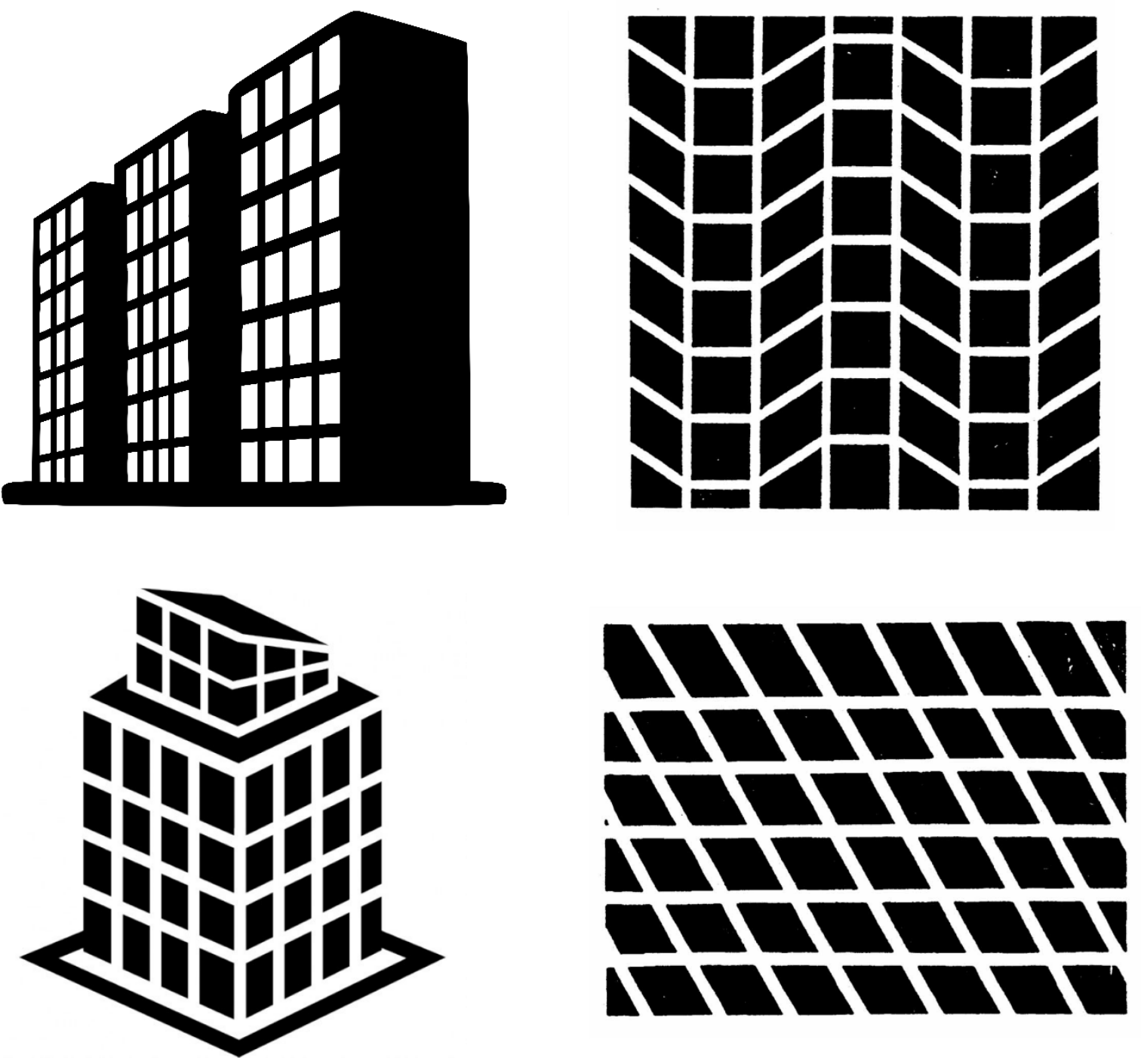

Figure 8. The above images instigate our familiar views, so we see illusion of blobs at the intersections of the grids (clockwise from left, $2^{\text {nd }}$ and $3^{\text {rd }}$ images have been taken from Spillmann, 1994). 

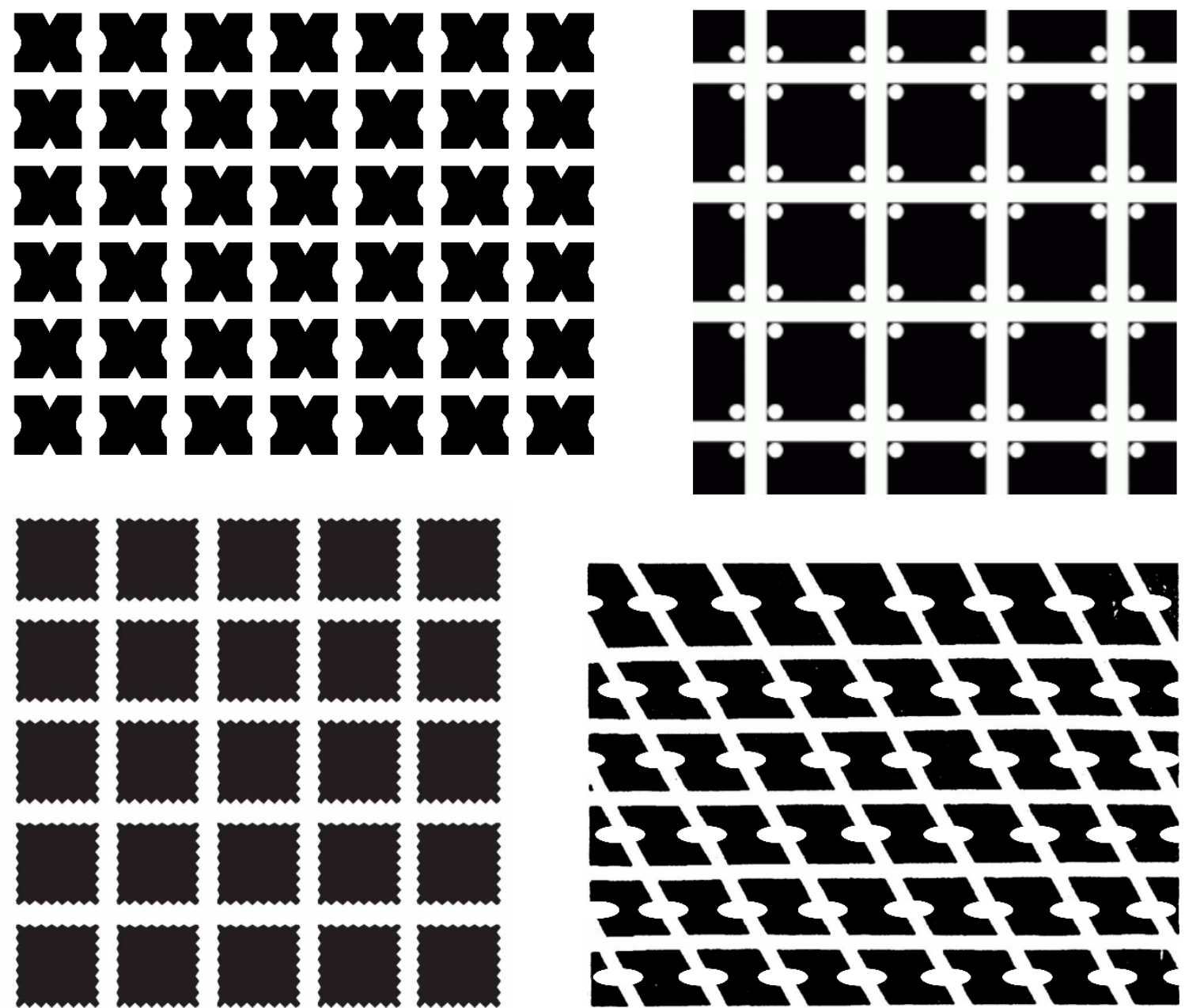

Figure 9. The above images do not coincide with our familiar views, so we do not see illusion of dark blobs at the intersections of the grids (clockwise from left, $1^{\text {st }}$ image has been modified from Geier, 2008; $2^{\text {nd }}$ image has been taken from www.colormancer.com with permission; and $4^{\text {th }}$ image has been taken from Schiller \& Carvey, 2005). 


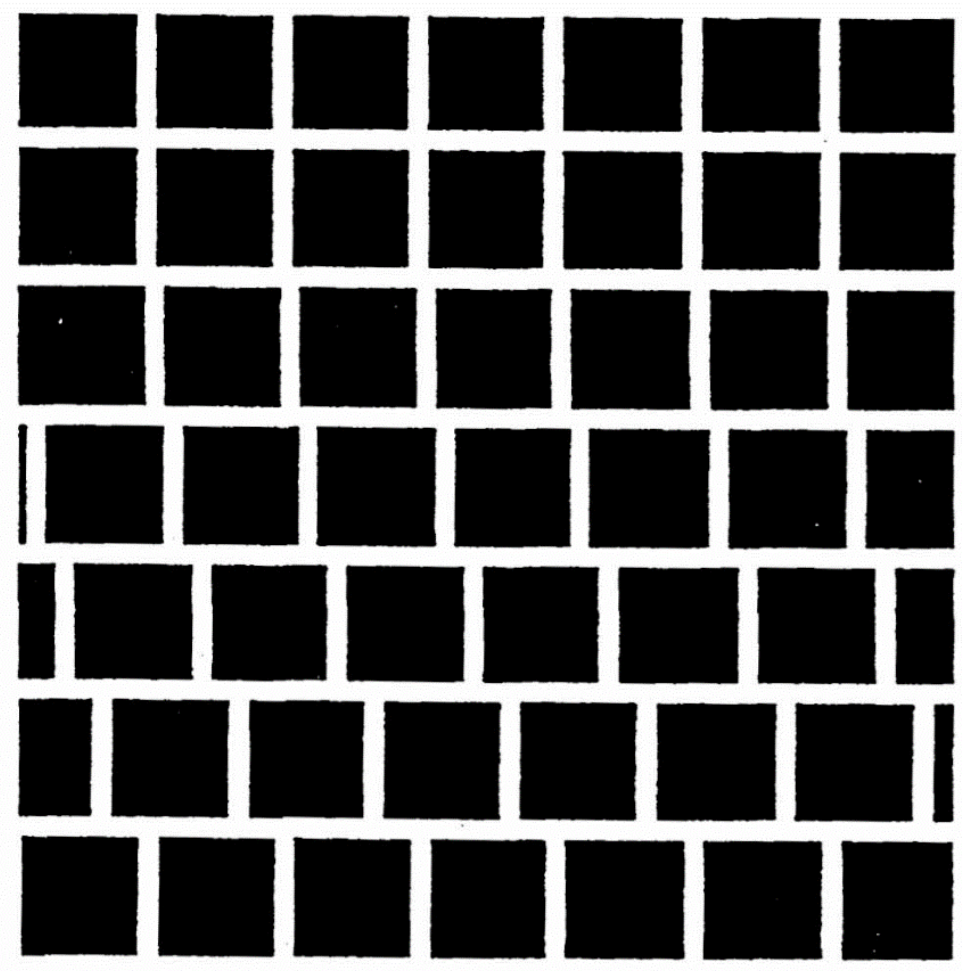

Figure 10. The upper half of the image coincides with our familiar view, and we see illusions at intersections. But as we progress downwards along the image, the view recedes from our familiarity; so along this, the illusions too gradually disappear (the image has been taken from Spillmann, 1994).

\section{Some Explanations of the Observations Regarding Hermann Grid Illusion:}

(1) Troscianko found that imposing more illumination on the intersections of the grid, nullification of the illusion is possible at a certain level. The reason is if we produce artificial glows at the intersections of a hollow Hermann grid, it will scatter hallows around instead of production of black smudges. Later on, in scintillating grid, Schrauf et al. put white discs on the darkened grid bars. In this case, we see scintillating dark spots at white discs, logic of which has already been described [Troscianko, 1982].

(2) Wolfe, through experiments on subjects, found that HGI is more conspicuous when the number of grid intersections are more. There are two reasons behind it. First, the greater number of intersections consolidate our familiarity with grid views more. And second, any optical illusion is more prominent at the peripheral vision as already described because of greater illusory coefficient at the peripheral visual field. So, with more intersections, we get more of grid at our peripheral visual field to let the illusion be generated more aggressively [Wolfe, 1984].

(3) Wist experimented with dark adaptation relating to HGI. In his experiment, when subjects observed the grid after dark adaptation, reaction time to experience the illusion increased 
[Wist, 1976]. Our photoreceptor cells have the ability to function over wide range of light intensity. Rods are extremely sensitive to light and responsible for night vision (scotopic vision). Cones are responsible for vision in bright light (photopic vision) and for color vision. Our perception of optical stimuli depends on the adaptive point on the light sensitivity scale. This adaptive point is settled by the differential activation and inhibition of photoreceptor cells of the retina. And we judge the outside visual world with respect to this adaptive point on a particular moment, that is it depends on what adaptive point our optical light sensitivity is fixed on at that moment. It is like whether $25^{\circ} \mathrm{C}$ is hot or cold that depends on what temperature you are living in [Das, 2017]. When we move from brighter to darker area or vice versa, there is a need for shifting the adaptive point first before we clearly see everything in the new context. And during this adaptation time, our upper visual mechanisms, that is translation of optical images to visual perception, remain comparatively dull. This is the reason behind increased reaction time in watching any illusion after dark adaptation. Here is to be mentioned that dark adaptation and light adaptation take different duration of times. And it should also be reiterated that for a particular color of light, illusory coefficient has different values for photopic and scotopic vision in a particular receptive field. In scotopic vision, the activities of cone cells are much reduced whereas there is increased production of rhodopsin from retinal and opsin till their supplies limit.

(4) Hermann grid illusion is visible also in case of contrasting chromatic grids (figure 11). But the illusion is not prominent always when the grid bars vary in colors, intensity, and overlapping [Schiller \& Carvey, 2005]. To successfully experience HGI, we have to consider two things must. First, the image should evoke the memory of a real grid astoundingly, no other competitive illusory outcome should be generated. Second, we should take how that grid would look in reality for its being reflected in illusory view.
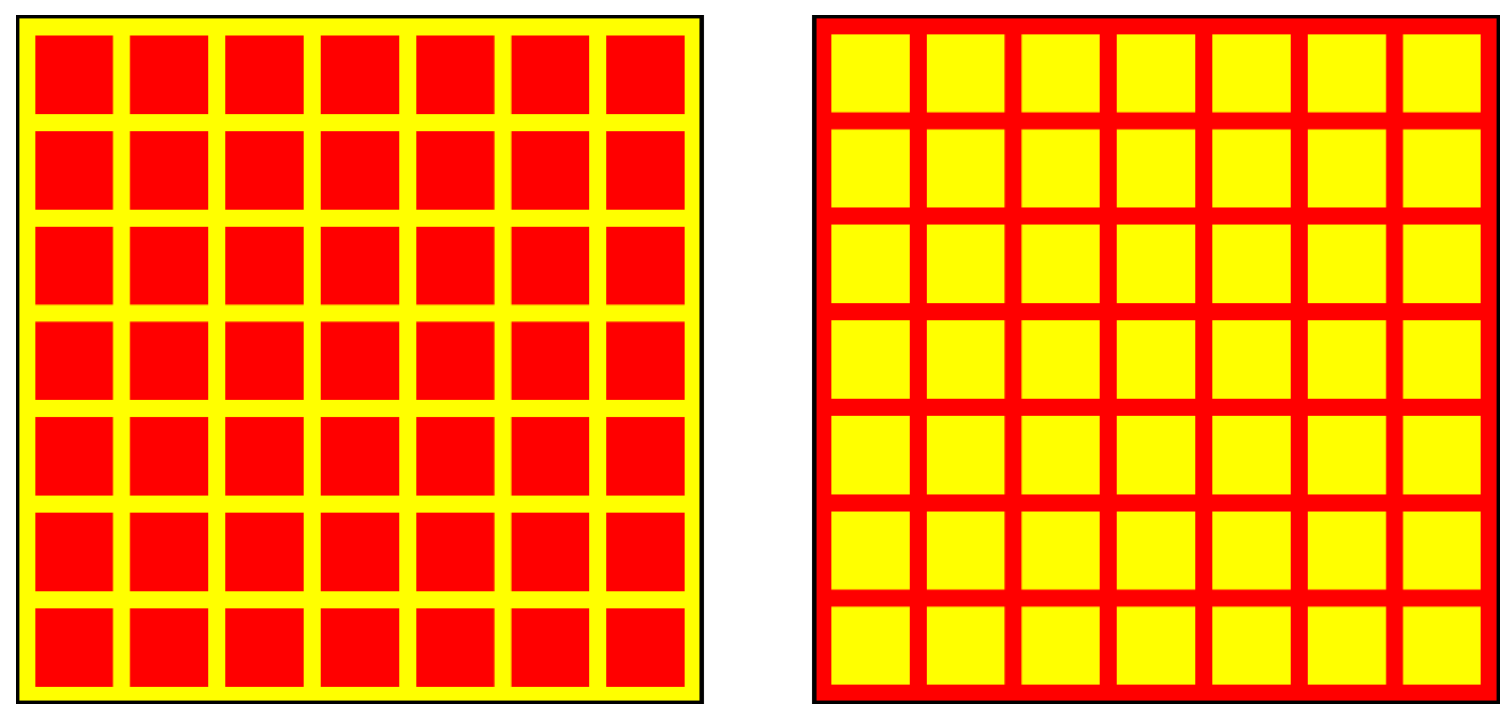

Figure 11. Hermann grid illusion on colored grids.

(5) Schiller \& Carvey (2005) presented two images (similar reported by Spillmann \& Levine, 1971) - one where low contrast bars were imposed on high contrast white bars against the background of black, and another where high contrast white bars were 
imposed on low contrast bars (figure 12). Here, we can see in the first case smudges at the intersections of the grid, but not in the second case. The reason behind it is lateral inhibition that what happens in Mach bands illusion, because the illusion is visible even in focal view and remains unchanged even when grids are tilted at $45^{\circ}$. In the first case, inhibition occurs at the intersections; in the second case, inhibition occurs on both sides of the white bars but as it is being divided it is not that prominent.

(6) Sometimes, we see fine lines running through the grid bars (more visible on white grid against black) even in distorted and rotated grids - which is particularly prominent on computer screen. That is also due to the effects of lateral inhibition. In case of white grid bars, due to 'off center on surround' effect of receptive fields, margins of the grid bars look whiter than the center.

To differentiate lateral inhibition and imaginary illusion, we should check whether it is visible at the fixation point (foveal view) or not through adjusting from different distances. As the effects of lateral inhibition is dependent on eccentricity and receptive field sizes, the view will also alter when watched from different distances from the object.

But here, I should wish to add few words. Lateral inhibition occurs in a single receptive field through off-on or on-off responses which is responsible for visual contrast (Hartline \& Graham, 1932). This is mediated through the inhibitory effects of the horizontal cells in outer plexiform layer, and through the reciprocal relation between hyperpolarized and depolarized bipolar cells in retina (Werblin \& Dowling, 1969). But lateral inhibition effectuates in the surrounding receptive fields also in declining degrees to some extent through horizontal cells and probably amacrine cells in inner plexiform layer in retina. Because if it had been effective in only single row of receptive fields along the contrast, the margin of lateral inhibition would have been sharp. Instead, the effects of it gradually diminishes to merge with the uniform view. However, much studies should be conducted. 

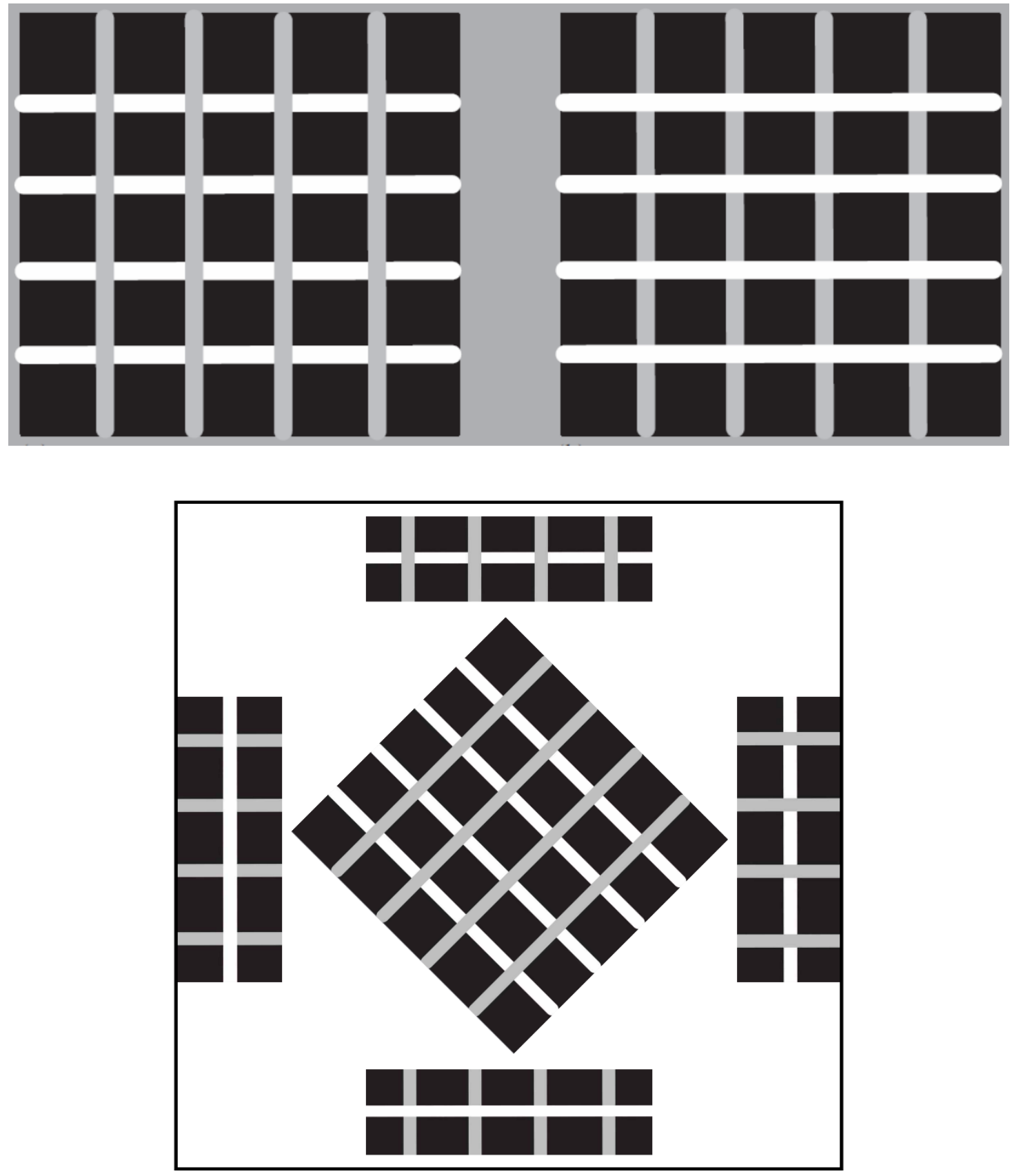

Figure 12. Effects of lateral inhibition on bars (images from Schiller \& Carvey, 2005).

\section{Conclusions:}

There are different explanations for Hermann Grid illusion that have been proposed by different authors along the time. But any of them cannot explain convincingly all the aspects of the Hermann Grid illusion and its deviations. This article has provided hopefully the most 
probable explanation of Hermann Grid optical illusion. Still there could be many other points which escaped in this article, that may necessitate further discussion. 


\section{References:}

1. Baumgartner G (1960). "Indirekte Grobenbestimmung der rezeptiven Felder der Retina beim Menschen mittels der Hermannschen Gittertauschung". Pflugers Archiv fur die gesamte Psychologie; 1960, 272: 21-22.

2. Bergen J R (1985). “Hermann's grid: new and improved (abstract)". Investigative Ophthalmology and Visual Science; supplement 26: 280.

3. Das K K (2017). "A Theoretical Approach to Define and Analyze Emotions". International Journal of Emergency Mental Health; 19(4): 374, 1-14.

4. Das K K (2018). "Role of pre-embedded associated memory in generation of optical illusions". Journal of psychology and cognition; 2018, 3(2): 37-49.

5. David S S J (1986). "Peripheral vision and child pedestrian accidents". Br J Psychol; 1986, 77(4): 433-450.

6. Ganong W F (2019). Ganong's Review of Medical Physiology. 26 $^{\text {th }}$ ed.; Editors: Barrett K

E, Barman S M, Brooks H L, Yuan J; 2019. McGraw-Hill Education.

7. Geier J (2008). "Straightness as the main factor of the Hermann grid illusion". Perception; 2008, 37(5): 651-665.

8. Hermann L (1870). "Eine Erscheinung des simultanen Contrastes". Pfliigers Archiv fur die gesamte Physiologies; 3: 13-15.

9. Hartline H K, Graham C H (1932). "Nerve impulses from single receptors in the eye". Journal of Cellular and Comparative Physiology; 1932, 1: 277-295.

10. Schrauf M, Lingelbach B, Wist E R (1997). "The Scintillating Grid Illusion". Vision Research; 1997, 37(8): 1033-1038.

11. Schiller P H, Carvey C E (2005). "The Hermann grid illusion revisited". Perception; 2005, 34: 1375-1397.

12. Sereno M I et al. (1995). "Borders of multiple visual areas in humans revealed by functional MRI”. 1995, Science; 268: 889-893.

13. Spillmann L (1994). " The Hermann grid illusion: a tool for studying human perceptive field organization". Perception; 1994, 23: 691-708.

14. Spillmann L, Levine J (1971). "Contrast enhancement in a Hermann grid with variable figure-ground ratio". Experimental Brain Research; 13: 547-559.

15. Troscianko T (1982). "A given visual field location has a wide range of perceptive field sizes". Vision Research; 22: 1363-1369. 
16. Werblin F S, Dowling J E (1969). "Organization of the retina of the mudpuppy, Necturus maculosus. II. Intracellular recording”. Journal of Neurophysiology; 1969, 32(3): 339-355.

17. Wist E R (1976). "Dark adaptation and the Hermann grid illusion". Perception \& Psychophysics; 20(1): 10-12.

18. Wolfe J M (1984). "Global factors in the Hermann grid illusion". Perception; 1984, 13: 33-40. 